\title{
Tim-3 is upregulated in human colorectal carcinoma and associated with tumor progression
}

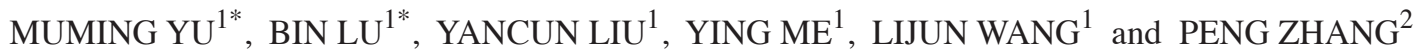 \\ ${ }^{1}$ Department of Emergency, Tianjin Medical University General Hospital, Tianjin 300071; ${ }^{2}$ School of \\ Basic Medical Sciences, Medical Institution of Peking University, Beijing 100191, P.R. China
}

Received August 27, 2015; Accepted August 16, 2016

DOI: $10.3892 / \mathrm{mmr} .2016 .6065$

\begin{abstract}
T cell immunoglobulin mucin-3 (Tim-3) has previously been implicated in the immune response and tumor biology. Colorectal carcinoma (CRC) is a malignancy, which is closely associated with inflammation. However, the role of Tim-3 in the progression of CRC remains to be fully elucidated. The present study aimed to investigate the role of Tim-3 in the progressive activities of human CRC. A total of 30 clinical CRC tissues and their adjacent tissues were collected. Slides from another 112 cases that underwent CRC surgical resection were also obtained. The protein and mRNA levels of Tim-3 in the clinical tissues and in CRC cell lines were initially examined using western blot and reverse transcription-quantitative polymerase chain reaction analyses, respectively. Immunohistochemical staining was performed to detect Tim-3 in the CRC samples. Specific small interfering (si)RNA against Tim-3 (siTim-3) was synthesized to knock down the expression of Tim-3, and the subsequent effects of Tim-3 knockdown on cell proliferation, migration and invasion were assessed. The data obtained showed that Tim-3 was expressed at high levels in the CRC tissues, compared with the non-cancerous tissues. The expression of Tim-3 in the clinical tissues was significantly associated with tumor size $(\mathrm{P}=0.007)$, tumor-node-metastasis staging $(\mathrm{P}<0.0001)$ and distant metastasis $(\mathrm{P}<0.0001)$. Knockdown of Tim-3 significantly reduced the cell proliferative rate of HCT116 and HT-29 cells. Wound closure activity was also inhibited by knockdown of Tim-3 in these two cell lines, and the migration and invasive abilities of these two cell lines were consistently decreased following knockdown of Tim-3. Taken together, Tim-3 was found to be a
\end{abstract}

Correspondence to: Dr Muming Yu, Department of Emergency, Tianjin Medical University General Hospital, 22 Qixiangtai Road, Heping, Tianjin 300071, P.R. China

E-mail: mumingyu1943@gmail.com

*Contributed equally

Key words: T cell immunoglobulin mucin-3, colorectal carcinoma, proliferation, migration, invasion critical mediator in the progression of CRC and may serve as a potential therapeutic target for the treatment of CRC.

\section{Introduction}

Colorectal carcinoma (CRC) is one of the most frequent malignancies affecting men and women worldwide. According to a previous statistic, a total of 102,480 new cases of CRC were estimated within the United States in 2013. In addition, 50,830 cases of CRC-associated mortality were estimated, making it the third leading cause of cancer-associated mortality worldwide (1). Although a level of progression has been achieved in treating $\mathrm{CRC}$ in past decades, the overall survival rate of patients suffering from CRC has remained poor $(2,3)$. Therefore, the identification of novel strategies for the treatment and prevention of CRC is urgently required.

Global evidence has established that inflammation is a well-recognized risk factor for cancer development (4-6). Anti-inflammatory agents have been shown to be associated with reduced risks of developing CRC and improved survival rates in patients with CRC $(7,8)$. Inflammation also has effects on tumor biology. For example, the local intratumoral inflammatory response, as evidenced by a high density of tumor-infiltrating lymphocytes, is considered to be a prognostic indicator for several types of malignancy, including CRC $(9,10)$. By contrast, systemic inflammation has always been associated with poor prognosis in CRC $(11,12)$. Therefore, the development and progression of CRC may be closely associated with immune regulatory processes.

T cell immunoglobulin mucin-3 (Tim-3) belongs to the Tim family, the members of which are cell surface receptors differentially expressed on mature $\mathrm{T}$ lymphocytes and macrophages. Specifically, Tim-3 is expressed in the Th1 subset, however, it is not expressed on Th2 cells $(13,14)$. The expression of Tim-3 is also present on macrophages, dendritic cells and mast cells $(15,16)$. The mechanisms underlying the immune regulatory reaction of Tim-3 are associated with controlling the functionality of T cell subsets, which occurs by inducing activating or apoptotic signals following interaction with its ligand, galectin-9 (17). Of note, with the exception of the immune response, increasing evidence has suggested that Tim-3 has functional roles in tumor biology. The expression of Tim-3 in peripheral blood monocytes and in tumor tissues has been suggested to be prognostic in prostate cancer (18). 
Tim-3 may affect development and progression, and be a therapeutic target in prostate cancer (19). The role of Tim-3 in human tumorigenesis is not limited to prostate cancer. Its tumor involvement in humans has been widely reported in various types of cancer, including clear cell renal cell carcinoma, hepatocellular carcinoma and melanoma (20-23). Targeting Tim-3 pathways has been suggested to reverse T cell exhaustion and restore antitumor immunity (24), therefore, Tim-3-targeted antitumor immunotherapy has been suggested as a prospective therapeutic strategy (21).

Despite the emerging evidence that Tim-3 may be critical in tumorigenesis, the role of Tim-3 in CRC remains to be fully elucidated. Considering CRC is closely associated with regulatory processes in inflammation, the present study hypothesized that Tim-3 may be a critical molecular involved in the development and progression of CRC. Therefore, the present study aimed to investigate whether Tim-3 is aberrantly expressed in clinical CRC samples and to assess the biological activities of Tim-3 in CRC cell lines.

\section{Materials and methods}

Human samples. Tissue samples from 30 cases of CRC and their adjacent non-cancerous tissues were collected from patients who underwent surgical tumor resection at Tianjin Medical University General Hospital (Tianjin, China) between January 1, 2014 and January 1, 2015. Slides from 112 paraffin-embedded CRC cases were also obtained from The Department of pathology, Tianjin Medical University General Hospital. All patients confirmed involvement in the present study, and written consent was obtained. This research was approved by the ethics committee of Tianjin Medical University General Hospital.

Histological and immunohistochemical (IHC) analysis. Following dissection from the patients, the tumor tissues were fixed in formaldehyde solution and embedded in paraffin to produce $4 \mu \mathrm{m}$ slices. Following antigen retrieval in $0.1 \mathrm{M}$ citric acid buffer ( $\mathrm{pH}$ 6.0) in a microwave, the slices were incubated with primary antibody against Tim-3 (cat. no. ab185703; Abcam, Cambridge, UK) at $4^{\circ} \mathrm{C}$ overnight. On the subsequent day, the slices were washed with Tris-buffered saline three times and incubated with secondary antibody (1:1,000; cat. no. sc-3836; Santa Cruz Biotechnology, Inc., Dallas, TX, USA) at $37^{\circ} \mathrm{C}$ for $1 \mathrm{~h}$, following which the slices were developed with $0.05 \%$ diaminobenzidine supplemented with $0.01 \%$ $\mathrm{H}_{2} \mathrm{O}_{2}$. As a negative control, normal goat serum (Beyotime Institute of Biotechnology, Haimen, China) was used in place of the specific primary antibody. Images were captured with a Nikon light microscope (Nikon, Tokyo, Japan; magnification, $\mathrm{x} 400)$.

Cell culture and antibodies. The human colorectal adenocarcinoma cell lines, COLO 205 and HT-29, the CRC cell line, HCT116 and the human embryonic kidney cell line, 293T, were purchased from America Type Culture Collection (Manassas, VA, USA). All cells were cultured in the Dulbecco's modified Eagle's medium (Gibco; Thermo Fisher Scientific, Inc. Waltham, MA, USA) supplied with $10 \%$ fetal bovine serum (FBS; Gibco; Thermo Fisher Scientific, Inc.) in a humidified incubator with $5 \% \mathrm{CO}_{2}$ at $37^{\circ} \mathrm{C}$. For the transfection assays, the cells were grown until $60 \%$ confluent and transfected with small interfering (si)RNAs using Lipofectamine 2000 (Invitrogen; Thermo Fisher Scientific, Inc.) according to the manufacturer's protocol. GAPDH was included as an internal control, and its primary antibody and secondary antibodies were purchased from Santa Cruz Biotechnology, Inc.

RNA isolation and reverse transcription-quantitative polymerase chain reaction ( $R T-q P C R)$ analysis. Total RNA from the human tissues and cultured cells were extracted using TRIzol solution (Takara Biotechnology Co., Ltd., Dalian, China) and quantified using a Nanodrop 2000 spectrophotometer (Thermo Fisher Scientific, Inc.). A $1 \mu \mathrm{g}$ sample of mRNA was reverse transcribed using PrimeScript RT Master Mix (Perfect Real Time) kit (Takara Biotechnology Co., Ltd.) and qPCR was performed in an ABI PRISM 7900 Real Time system (Applied Biosystems; Thermo Fisher Scientific, Inc.) using the SYBR Premix Ex Taq kit (Takara Biotechnology Co., Ltd.). The primers used were as follows: Tim-3, forward 5'-GCTACTACT TACAAGGTCCTCAG-3' and reverse 5'-ATTCACATCCCTTTCATCAGTC-3'; GAPDH, forward 5'-GTGGACATCCGCAAAGAC-3' and reverse 5'-AAAGGG TGTAACGCAACTA-3'. U Initial denaturation was performed at $95^{\circ} \mathrm{C}$ for $30 \mathrm{sec}$, and PCR by 40 cycles of $95^{\circ} \mathrm{C}$ for $5 \mathrm{sec}$ and $60^{\circ} \mathrm{C}$ for $35 \mathrm{sec}$. All experiments were performed in triplicate at least three times. Values were calculated used the $2^{-\Delta \Delta \mathrm{Cq}}$ method (25)

Western blot analysis. The cells were lysed with lysis buffer supplemented with protease inhibitors. The proteins obtained from the CRC cell lines were quantified using a Bicinchoninic Acid kit (Thermo Fisher Scientific, Inc.). Subsequently, a total of $50 \mu \mathrm{g}$ protein was loaded onto a $10 \%$ SDS-PAGE gel for separation, and then transferred onto a nitrocellulose membrane. Following blocking in 5\% milk for $1 \mathrm{~h}$ at room temperature, the membrane was incubated with primary antibodies against Tim-3 (1:1,000; cat. no. ab185703; Abcam) and GAPDH (1:1,000; cat. no. sc-365062; Santa Cruz Biotechnology, Inc.) at $4{ }^{\circ} \mathrm{C}$ overnight. The following day, the membrane was washed with TBS with Tween 20 three times and incubated with secondary antibodies (1:1,000; cat. no. sc-3836; Santa Cruz Biotechnology, Inc.) for another $1 \mathrm{~h}$ at $37^{\circ} \mathrm{C}$. Protein expression was quantified using enhanced chemiluminescence (Thermo Fisher Scientific, Inc.) and images were captured using the LAS3000 imaging system (Fujifilm Corporation, Tokyo, Japan).

Cell viability assay. The HCT116 and HT-29 cells were seeded in 96 -well plates $\left(5 \times 10^{3}\right.$ cells/well) and allowed to grow overnight at $37^{\circ} \mathrm{C}$. The cells were then transfected with either control siRNA or specific siRNA against Tim-3 (synthesized by GenePharma Co., Ltd., Shanghai, China) and grown for another $72 \mathrm{~h}$. Subsequently, cell viability assays were performed consecutively for 5 days using MTT solution. Following the addition of $2 \mathrm{mg} / \mathrm{ml} \mathrm{MTT}$ for $4 \mathrm{~h}$ at $37^{\circ} \mathrm{C}$, the medium was discarded and $200 \mu \mathrm{l}$ of DMSO was added to each well. The solution was added into each well on each of the monitored days. The cells were incubated for an additional $5 \mathrm{~min}$ on a shaker and the optical density was determined at $570 \mathrm{~nm}$. 
Table I. Correlation of the expression of Tim-3 with clinicopathological parameters in 112 cases of colorectal carcinoma.

\begin{tabular}{|c|c|c|c|c|c|}
\hline \multirow[b]{2}{*}{ Parameter } & \multirow[b]{2}{*}{ No. } & \multicolumn{2}{|c|}{ Expression of Tim-3 } & \multirow[b]{2}{*}{ P-value } & \multirow[b]{2}{*}{$\begin{array}{l}\text { Correlation } \\
\text { coefficient }\end{array}$} \\
\hline & & $\begin{array}{c}\text { Low } \\
(n=65)\end{array}$ & $\begin{array}{l}\text { High } \\
(n=47)\end{array}$ & & \\
\hline Age (years) & & & & 0.220 & \\
\hline$<50$ & 29 & 14 & 15 & & -0.117 \\
\hline$\geq 50$ & 83 & 51 & 32 & & \\
\hline Gender & & & & 0.775 & \\
\hline Male & 68 & 43 & 25 & & -0.027 \\
\hline Female & 44 & 29 & 15 & & \\
\hline Tumor size (cm) & & & & 0.007 & \\
\hline$\leq 2$ & 71 & 54 & 27 & & 0.258 \\
\hline $2-5$ & 29 & 11 & 18 & & \\
\hline$>5$ & 2 & 0 & 2 & & \\
\hline TNM stage & & & & $<0.001$ & \\
\hline I and II & 85 & 58 & 27 & & 0.367 \\
\hline III and IV & 27 & 7 & 20 & & \\
\hline Distant metastasis & & & & $<0.001$ & \\
\hline No & 93 & 61 & 32 & & 0.339 \\
\hline Yes & 19 & 4 & 15 & & \\
\hline
\end{tabular}

Tim-3, T cell immunoglobulin mucin-3; TNM, tumor-node-metastasis.

Cell migration and invasion assays. The HCT116 and HT-29 cells were cultured in 24-well plates and transfected with the specific Tim-3 siRNA or control siRNA. At $48 \mathrm{~h}$ post-transfection, the cells were harvested in serum-free medium as a single cell suspension, and $150 \mu \mathrm{l}$ volume of cell suspension $\left(3 \times 10^{4}\right.$ cells) was seeded into the upper Transwell chamber (Corning Incorporated, Corning, NY, USA). A $600 \mu \mathrm{l}$ volume of medium supplemented with $10 \%$ FBS was added to the lower chamber. For the invasion assay, the chamber was coated with Matrigel and incubated for $6 \mathrm{~h}$ at $37^{\circ} \mathrm{C}$ to solidify prior to seeding the cells into the chamber. Subsequently, the cells were incubated for $24 \mathrm{~h}$, then, fixed with ice-cold methanol for $20 \mathrm{~min}$ and then stained with $0.1 \%$ crystal violet for another $5 \mathrm{~min}$. Images were captured under a Nikon light microscope (magnification, x200). The number of cells from each well were counted in 10 randomly selected fields.

Wound healing assay. The HCT116 and HT-29 CRC cells $\left(1 \times 10^{6}\right)$ were seeded into 6 -well plates and transfected with specific siRNA against Tim-3 (0, 0.3, 0.6, 0.9, 1.2 and $1.5 \mu \mathrm{m})$. At $48 \mathrm{~h}$ post-transfection, the cells were washed twice with PBS and a $10 \mu l$ sterile pipette tip was then used to scratch a cross in the center of each well. Following scratching, the cells were rinsed with PBS again, and immediately placed in serum-free medium. The cells were then allowed to migrate for another $24 \mathrm{~h}$, following which the scratches in each group were observed and images were captured. Each assay was repeated in triplicate at least three times.

Statistical analysis. SPSS software (Chicago, IL, USA) was used for statistical analysis. Student's $t$-test was used for simple comparisons between different groups. Regression analysis was used to evaluate dose-response associations. Values are presented as the mean \pm standard deviation. $\mathrm{P}<0.05$ was considered to indicate a statistically significant difference.

\section{Results}

Tim-3 is overexpressed in CRC and associated with tumor progression. The present study first investigated the expression levels of Tim-3 in 30 clinical CRC tissues and their adjacent non-cancerous tissues. The protein levels were analyzed using western blot analysis. It was shown that the protein levels of Tim-3 in the cancerous samples were significantly higher, compared with those in the paired non-cancerous samples in the representative four cases (Fig. 1A). The total RNA extracted from the CRC tissues and their adjacent non-cancerous tissues were subjected to RT-qPCR analysis. It was observed that the mRNA levels of Tim-3 in the CRC tissues were $>5$-fold higher than those in the adjacent tissues (Fig. 1B). These results suggested that Tim-3 was expressed at a high level in the clinical CRC tissues. Furthermore, to assess the association between the clinical expression of Tim-3 and clinicopathological parameters, IHC staining was performed to detect the Tim- 3 antigen in the slides from the 112 CRC cases. Based on the IHC results, the staining intensity of Tim-3 was classified as low expression or high expression for each case (Fig. 1C). Statistical analysis revealed that the expression levels of Tim-3 were significantly positively correlated with tumor size $\left(\mathrm{P}=0.007 ; \mathrm{R}^{2}=0.258\right)$, TNM staging $\left(\mathrm{P}<0.001 ; \mathrm{R}^{2}=0.367\right)$ and distant metastasis $\left(\mathrm{P}<0.001 ; \mathrm{R}^{2}=0.339\right)$, however, expression was not correlated with demographic data, including age and 

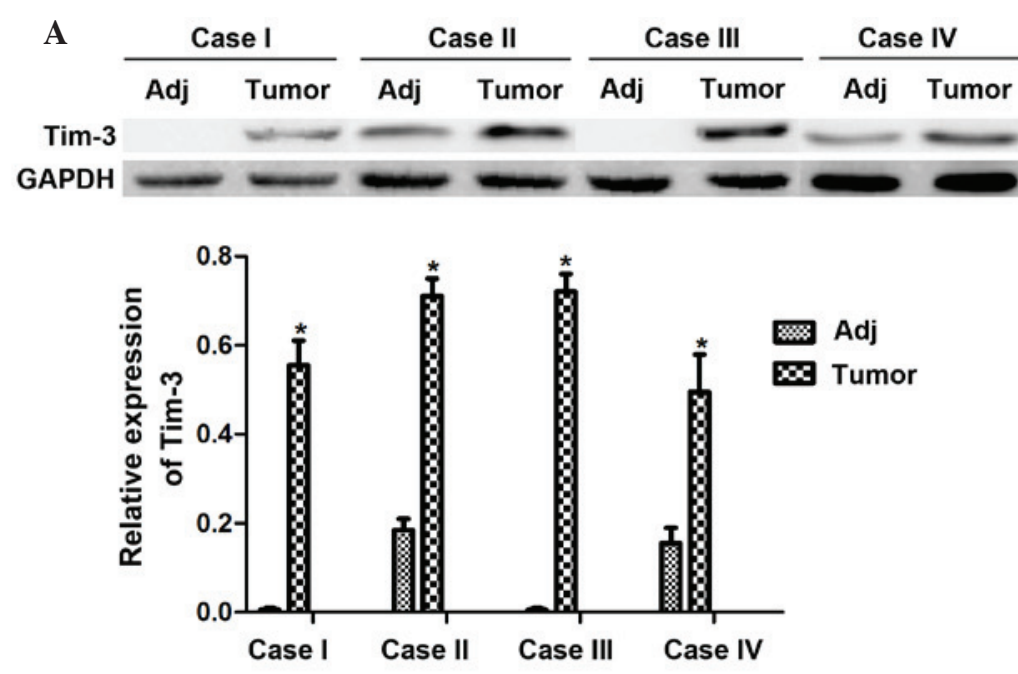

B

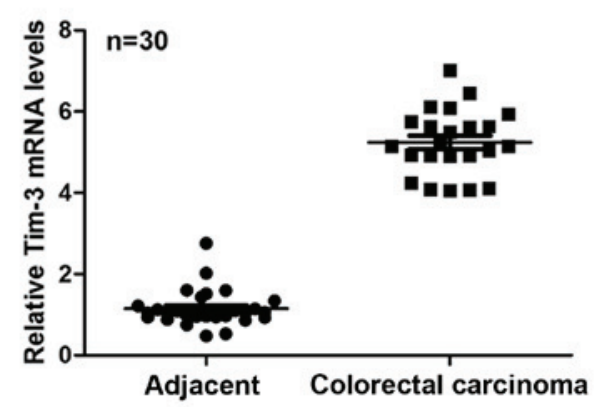

D

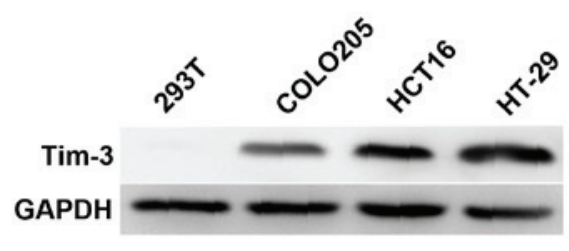

Figure 1. Tim-3 is overexpressed in CRC tissues and in cultured CRC cell lines. Tissues from 30 patients with CRC were dissected and used to extract total RNA and proteins. (A) Western blot analysis revealed that the protein levels of Tim-3 were significantly higher in the clinical CRC samples, compared with the paired non-cancerous samples. GAPDH was included as an inner control. ${ }^{*} \mathrm{P}<0.05$. (B) Total RNAs from the 30 clinical CRC samples and adjacent tissues were subjected to reverse transcription-quantitative polymerase chain reaction analysis. It was shown that the mRNA levels of Tim-3 were higher in the CRC tissues, compared with the adjacent non-cancerous tissues. (C) Immunohistochemical analysis was performed in slides from 112 clinical cases of CRC. For each case, the staining intensity of Tim-3 was classified as low (upper panel) or high (lower panel) expression (magnificantion, x400). (D) Differential expression of Tim-3 was shown in CRC cell lines. The 293T cell line was included as a control. Tim-3, T cell immunoglobulin mucin-3; CRC, colorectal carcinoma; Adj, adjacent non-cancerous tissue.

gender (Table I). This data suggested that the expression of Tim-3 is associated with tumor progression in CRC. In addition, COLO 205 and HT-29 are two representative human CRC cell lines, and HCT116 is a typical CRC cell line. Proteins from these cell lines were extracted to examine the expression levels of Tim-3 in vitro. Compared with the 293T control cells, Tim-3 was overexpressed in the CRC cell lines, particularly in the HCT116 and HT-29 cells (Fig. 1D). Therefore, these two cell lines were selected for subsequent analyses. These data suggested the close association between the expression of Tim-3 and oncogenic activity in CRC.

Knockdown of Tim-3 with specific siRNA in cultured CRC cells. CRC is a common malignancy worldwide and has high rates of metastasis. The present study aimed to assess whether Tim-3 is involved in the progressive activities of CRC. Specific siRNA against Tim-3 (siTim-3) was designed to knock down the expression of Tim-3 in cultured CRC cell lines. At $72 \mathrm{~h}$ post-transfection, the RNAs and proteins were extracted and subjected to RT-qPCR and western blot analyses, respectively. As shown in Fig. 2A, the mRNA levels of Tim-3 in the HCT116 and HT-29 cells were significantly decreased by siTim- 3 by up to $\sim 50 \%$, compared with the control groups. The protein levels of Tim-3 were also markedly decreased when the two cell lines were transfected with siTim-3 (Fig. 2B). These results revealed the high specificity of siRNA and the efficiency of transfection.

Knockdown of Tim-3 suppresses cell proliferation in CRC cells. The present study performed an MTT assay to examine the role of Tim-3 on cell proliferation in the HCT116 and HT-29 CRC cell lines. As shown in Fig. 3A, no significant differences were observed between the HCT116 cells of the three groups in the first 3 days. However, on day 4 , the proliferation rate was decreased by $18 \%$ in the siTim-3-treated group, whereas the control siRNA-transfected group remained stable. The suppression was more marked on day 5 by up to $25 \%$. Similar results were observed in the HT-29 cells, in which cell proliferation rate was decreased by $19 \%$ on day 4 and $31.25 \%$ on day 5 by siTim-3 (Fig. 3B). These data suggested that the knockdown of Tim-3 inhibited the proliferative activity of the HCT116 and HT-29 CRC cell lines.

Knockdown of Tim-3 inhibits cell migration and invasion in CRC cells. The present study further investigated the effects of Tim-3 knockdown on cell migration and invasion in vitro. Prior to the wound healing assay and Transwell assays, the HCT116 and HT-29 cells were transfected with either control siRNA 
A

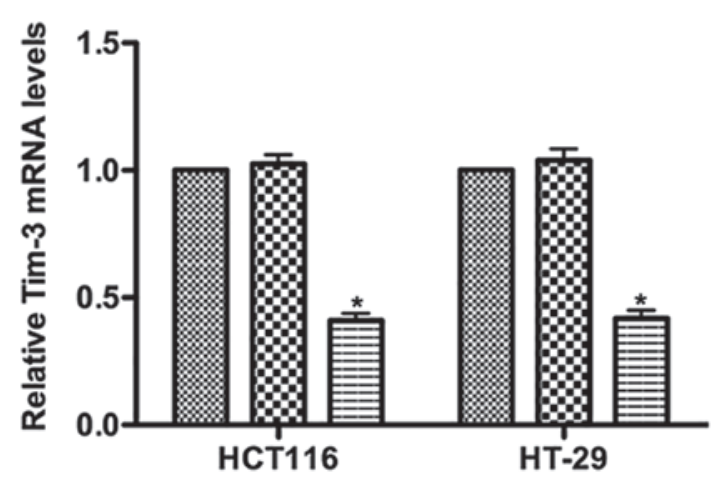

B

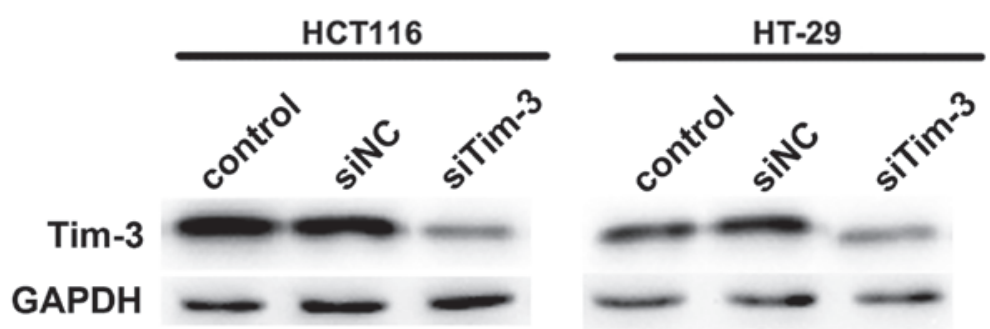

Figure 2. Knockdown of Tim-3 with specific siRNA in cultured colorectal carcinoma cells. (A) Reverse transcription-quantitative polymerase chain reaction analysis revealed that the mRNA levels of Tim- 3 were suppressed by $>50 \%$ by the specific siRNA in the HCT116 and HT-29 cell lines. ${ }^{*}<0.05$, vs. control. (B) Western blot analysis revealed that the protein levels of Tim-3 in the siTim-3-treated groups were significantly decreased, compared with those in the control groups. Each experiment was repeated in triplicate at least three times. Tim-3, T cell immunoglobulin mucin-3; siTim-3, specific small interfering RNA against Tim-3; NC, negative control.

A

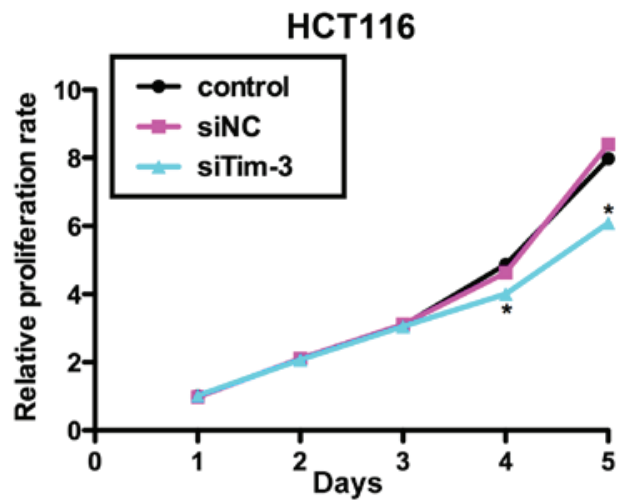

B

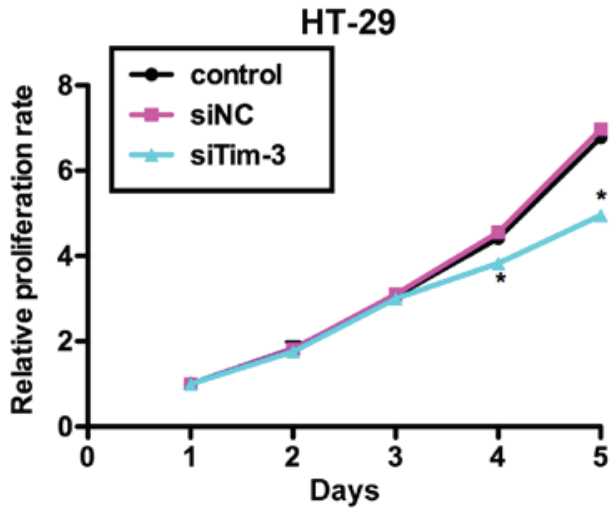

Figure 3. Knockdown of Tim-3 suppresses proliferation of colorectal carcinoma cells. Cells were transfected with siNC or siTim-3, grown for $72 \mathrm{~h}$ at $37^{\circ} \mathrm{C}$ and mixed with 3-(4,5-Dimethylthiazol-2-yl)-2,5-dipheny ltetrazoliumbromide methyl thiazolyl tetrazolium solution. Relative proliferation rates of (A) HCT116 cells and (B) HT-29 cells in different treatment groups are shown. "P $<0.05$, vs. control. Tim-3, T cell immunoglobulin mucin-3; siTim-3, specific small interfering RNA against Tim-3; NC, negative control. or various concentrations of siTim-3. As shown in Fig. 4A, no notable differences were observed between the control groups. However, in the siTim-3 groups, the rates of wound closure were significantly decreased in the two cell lines and these inhibitory effects were dose-dependent. The results of the Transwell assay confirmed the above observations. In the siTim-3-transfected HCT116 cells, the percentage of cells found to migrate to the inferior surface of the membrane were only 55 and $45 \%$ of the control groups in the migration and invasion assays, respectively. The HT-29 cells also exhibited lower migration and invasion rates when transfected with siTim-3, compared with the control groups (Fig. 4B and C). These results revealed that the knockdown of Tim-3 decreased the migration and invasion abilities of the cells in vitro.

\section{Discussion}

CRC is the third leading cause of cancer-associated mortality each year. Surgery and 5-fluorouracil-based adjuvant chemotherapy are recommended for patients with high-risk stage II and stage III CRC (26). However, the prognosis of CRC has remained poor over past decades. Previous ivestigations have predominantly focused on the immunotherapy of cancer, particularly those closely associated with inflammation (21). However, despite the wide recognition of CRC as an inflammation-associated malignancy, progression in immunotherapy has not been substantial in treating CRC until now.

The present study is the first, to the best of our knowledge, to report that Tim-3 is critical in CRC cell proliferation, migration and invasion. Tim-3 is an immune regulatory molecule, which triggers downstream cascade events upon stimulation 
A

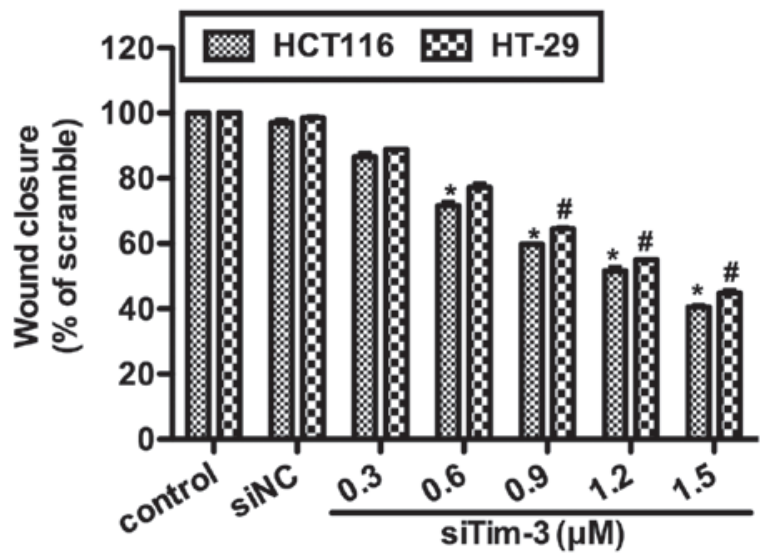

B

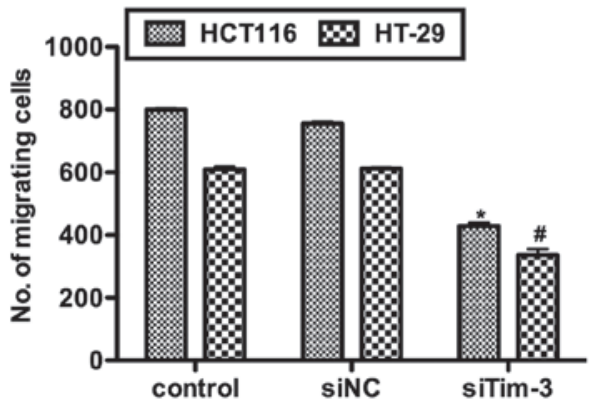

C

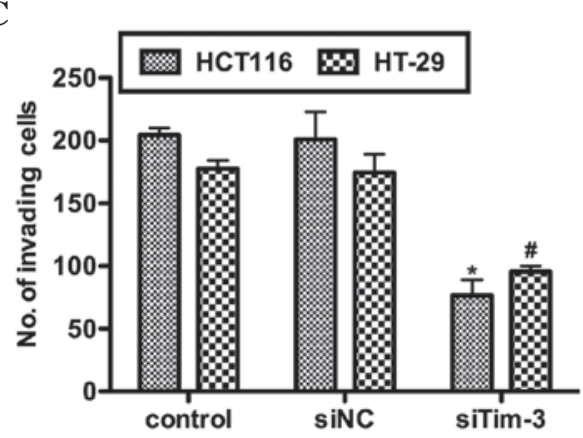

Figure 4. Tim-3 promotes cell migration and invasion in colorectal carcinoma cells. (A) HCT116 and HT-29 cells were transfected with control siRNA or different doses of tim-3 siRNA. The proportions of the wound were measured in each separate experiment and the percentage of wound closure in each treatment group was calculated. (B) Cell migration assay for HCT116 and HT-29 cells. (C) Cell invasion assay for HCT116 and HT-29 cells. The number of cells migrated to the inferior surface of the membrane was calculated in each group. ${ }^{*} \mathrm{P}<0.05$, vs. control in HCT116 cells, ${ }^{*} \mathrm{P}<0.05$, vs. control in HT-29 cells. Tim- 3 , T cell immunoglobulin mucin-3; siTim-3, specific small interfering RNA against Tim-3; NC, negative control.

by its ligand, galectin-9. Emerging evidence has demonstrated the importance of Tim-3 in human tumorigenesis. However, no studies have been performed to examine the role of Tim-3 in CRC. A previous study reported the expression profile of Tim-3 in pediatric Crohn's disease, in which Tim-3 was expressed at high levels in tissue samples of Crohn's disease and its expression was correlated with the pediatric Crohn's disease activity index (27). This report is important as Crohn's disease is widely-recognized to trigger an immune response and progress to $\mathrm{CRC}$ if not controlled. However, no further investigations have been performed with respect to the role of Tim-3 in CRC.

Tim-3 has previously been reported to be expressed at high levels in prostate cancer, hepatocellular carcinoma, renal cell carcinoma and melanoma $(18-20,22,23)$. In line with these reports, the present study found that the expression of Tim-3 was significantly higher in CRC cancerous samples, compared with adjacent non-cancerous samples. Of note, following the knockdown of Tim-3 with specific siRNA, it was found that cell proliferation was significantly inhibited whereas the proliferation rates of the control cells were unaffected. Wound healing abilities, which reflect cell migration potential, were also inhibited in two CRC cell lines, in a dose-dependent manner, and their migration and invasion abilities were also inhibited, as determined using Transwell assays. These in vitro results confirmed the results in vivo that the expression of Tim-3 was statistically associated with tumor TNM staging, distant metastasis and tumor size (Table I). However, these observations also suggested that Tim-3 promoted CRC cell oncogenic activities, including proliferation, migration and invasion, and confirmed the that Tim-3-targeted therapy may be anti-neoplastic (21).

The mechanism underlying Tim-3-mediated tumor progression remains to be fully elucidated. According to previous data, the interleukin-6 (IL-6)-signal transducer and activator of transcription (STAT)3 pathway is critical in tumor growth and metastasis in human hepatocellular carcinoma (28). DNA damage induces the IL-STAT3 pathway which has growth-promoting effects in human tumors (29). Inhibiting IL-6 reverses the Tim-3-mediated effects on HCC cell growth in vitro (23). Therefore, the IL-STAT3 pathway may be critical in the biological activities of Tim-3. However, another previous report demonstrated that the mechanisms involving the biological activities of Tim-3 may be distinct in different scenarios. It was observed that the suppression of downstream GATA binding protein 3 (GATA3) was an important mechanism by which Tim-3 triggered metastasis in renal cell carcinoma. However, distinct from inhibiting Tim-3, the same report documented that GATA3 was activated by Tim-3 in facilitating systemic lupus erythematosus. Therefore, Tim-3 may exert disease-promoting effects through inconsistent pathways. The mechanisms underlying the Tim-3-mediated progression of CRC may also be unique and require further investigation in the future.

In conclusion, the present study was the first to investigate the expression and involvement of Tim-3 in the progression of human CRC. Tim-3 was expressed at high levels in CRC tissues. The knockdown of Tim-3 significantly reduced cell proliferation, 
migration and invasion, and these results were consistent with those from the clinical tissues. Therefore, interference of Tim-3 may offer potential in CRC therapy. However, further investigations are required to reveal the detailed mechanisms.

\section{Acknowledgements}

The authors would like to thank Dr Peng Zhang for his professional technical support during the IHC assay.

\section{References}

1. Siegel R, Naishadham D and Jemal A: Cancer statistics, 2013. CA Cancer J Clin 63: 11-30, 2013.

2. Chen C, Wang L, Liao Q, Huang Y, Ye H, Chen F, Xu L, Ye M and Duan S: Hypermethylation of EDNRB promoter contributes to the risk of colorectal cancer. Diagn Pathol 8: 199, 2013.

3. Qu YL, Wang HF, Sun ZQ, Tang Y, Han XN, Yu XB and Liu K Up-regulated miR-155-5p promotes cell proliferation, invasion and metastasis in colorectal carcinoma. Int J Clin Exp Pathol 8: 6988-6994, 2015.

4. Diakos CI, Charles KA, McMillan DC and Clarke SJ Cancer-related inflammation and treatment effectiveness. Lancet Oncol 15: e493-e503, 2014.

5. Grivennikov SI, Greten FR and Karin M: Immunity, inflammation, and cancer. Cell 140: 883-899, 2010

6. Coussens LM and Werb Z: Inflammation and cancer. Nature 420 : 860-867, 2002

7. Cooper K, Squires H, Carroll C, Papaioannou D, Booth A, Logan RF, Maguire C, Hind D and Tappenden P: Chemoprevention of colorectal cancer: Systematic review and economic evaluation. Health Technol Assess 14: 1-206, 2010.

8. Goh CH, Leong WQ, Chew MH, Pan YS, Tony LK, Chew L, Tan IB, Toh HC, Tang CL, Fu WP and Chia WK: Post-operative aspirin use and colorectal cancer-specific survival in patients with stage I-III colorectal cancer. Anticancer Res 34: 7407-7414, 2014.

9. Pagès F, Berger A, Camus M, Sanchez-Cabo F, Costes A, Molidor R, Mlecnik B, Kirilovsky A, Nilsson M, Damotte D, et al Effector memory $\mathrm{T}$ cells, early metastasis, and survival in colorectal cancer. N Engl J Med 353: 2654-2666, 2005.

10. Mlecnik B, Tosolini M, Kirilovsky A, Berger A, Bindea G, Meatchi T, Bruneval P, Trajanoski Z, Fridman WH, Pagès F and Galon J: Histopathologic-based prognostic factors of colorectal cancers are associated with the state of the local immune reaction. J Clin Oncol 29: 610-618, 2011.

11. Canna K, McMillan DC, McKee RF, McNicol AM, Horgan PG and McArdle CS: Evaluation of a cumulative prognostic score based on the systemic inflammatory response in patients undergoing potentially curative surgery for colorectal cancer. $\mathrm{Br}$ J Cancer 90: 1707-1709, 2004.

12. Guthrie GJ, Roxburgh CS, Farhan-Alanie OM, Horgan PG and McMillan DC: Comparison of the prognostic value of longitudinal measurements of systemic inflammation in patients undergoing curative resection of colorectal cancer. Br J Cancer 109: 24-28, 2013.

13. Sabatos CA, Chakravarti S, Cha E, Schubart A, Sánchez-Fueyo A, Zheng XX, Coyle AJ, Strom TB, Freeman GJ and Kuchroo VK: Interaction of Tim-3 and Tim-3 ligand regulates T helper type 1 responses and induction of peripheral tolerance. Nat Immunol 4 $1102-1110,2003$
14. Monney L, Sabatos CA, Gaglia JL, Ryu A, Waldner H, Chernova T, Manning S, Greenfield EA, Coyle AJ, Sobel RA, et al: Th1-specific cell surface protein Tim-3 regulates macrophage activation and severity of an autoimmune disease. Nature 415: 536-541, 2002.

15. Horlad H, Ohnishi K, Ma C, Fujiwara Y, Niino D, Ohshima K, Jinushi M, Matsuoka M, Takeya M and Komohara Y: TIM-3 expression in lymphoma cells predicts chemoresistance in patients with adult T-cell leukemia/lymphoma. Oncol Lett 12: 1519-1524, 2016.

16. Liu J, Zhang S, Hu Y, Yang Z, Li J, Liu X, Deng L, Wang Y, Zhang X, Jiang T and Lu X: Targeting PD-1 and Tim-3 pathways to reverse CD8 T-cell exhaustion and enhance ex vivo T-cell responses to autologous dendritic/tumor vaccines. J Immunother 39: 171-180, 2016.

17. Kuchroo VK, Umetsu DT, DeKruyff RH and Freeman GJ: The TIM gene family: Emerging roles in immunity and disease. Nat Rev Immunol 3: 454-462, 2003.

18. Piao YR, Piao LZ, Zhu LH, Jin ZH and Dong XZ: Prognostic value of $T$ cell immunoglobulin mucin-3 in prostate cancer. Asian Pac J Cancer Prev 14: 3897-3901, 2013.

19. Piao YR, Jin ZH, Yuan KC and Jin XS: Analysis of Tim-3 as a therapeutic target in prostate cancer. Tumour Biol 35: 11409-11414, 2014

20. Zheng H, Guo X, Tian Q, Li H and Zhu Y: Distinct role of Tim-3 in systemic lupus erythematosus and clear cell renal cell carcinoma. Int J Clin Exp Med 8: 7029-7038, 2015.

21. Ngiow SF, Teng MW and Smyth MJ: Prospects for TIM3-targeted antitumor immunotherapy. Cancer Res 71: 6567-6571, 2011

22. Wiener Z, Kohalmi B, Pocza P, Jeager J, Tolgyesi G, Toth S, Gorbe E, Papp Z and Falus A: TIM-3 is expressed in melanoma cells and is upregulated in TGF-beta stimulated mast cells. J Invest Dermatol 127: 906-914, 2007.

23. Yan W, Liu X, Ma H, Zhang H, Song X, Gao L, Liang X and Ma C: Tim-3 fosters HCC development by enhancing TGF- $\beta$-mediated alternative activation of macrophages. Gut 64 : 1593-1604, 2015.

24. Sakuishi K, Apetoh L, Sullivan JM, Blazar BR, Kuchroo VK and Anderson AC: Targeting Tim-3 and PD-1 pathways to reverse T cell exhaustion and restore anti-tumor immunity. J Exp Med 207: 2187-2194, 2010.

25. Chono H, Saito N, Tsuda H, Shibata H, Ageyama N, Terao K, Yasutomi Y, Mineno J and Kato I: In vivo safety and persistence of endoribonuclease gene-transduced CD4+ T cells in cynomolgus macaques for HIV-1 gene therapy model. PLoS One 6: e23585, 2011

26. Smolle MA, Pichler M, Haybaeck J and Gerger A: Genetic markers of recurrence in colorectal cancer. Pharmacogenomics 16: 1315-1328, 2015.

27. Kim MJ, Lee WY and Choe YH: Expression of TIM-3, human $\beta$-defensin-2, and FOXP3 and correlation with disease activity in pediatric crohn's disease with infliximab therapy. Gut Liver 9: 370-380, 2015.

28. Yang X, Liang L, Zhang XF, Jia HL, Qin Y, Zhu XC, Gao XM, Qiao P, Zheng Y, Sheng YY, et al: MicroRNA-26a suppresses tumor growth and metastasis of human hepatocellular carcinoma by targeting interleukin-6-Stat3 pathway. Hepatology 58: 158-170, 2013.

29. Yun UJ,Park SE, Jo YS, Kim J and Shin DY: DNA damage induces the IL-6/STAT3 signaling pathway, which has anti-senescence and growth-promoting functions in human tumors. Cancer Lett 323: 155-160, 2012. 